\title{
Segregação ocupacional e diferenciais de renda por gênero e raça no Brasil: uma análise de grupos etários
}

\author{
Leonardo Souza Silveira* \\ Natália Leão Siqueira*
}

Nos últimos 30 anos, o Brasil consolidou aspectos da mudança estrutural no mercado de trabalho referentes à modernização ocupacional, participação feminina e expansão do ensino superior. Nesse sentido, coortes mais jovens se inserem em um contexto distinto daqueles presenciados em décadas passadas. Logo, este artigo tem como objetivo medir a variação da segregação ocupacional e a disparidade salarial por gênero e raça em três grupos etários. Assim, questiona-se: a segregação ocupacional por gênero e raça é menor entre os mais jovens? Isso se reflete em menor desigualdade salarial? Tais perguntas são analisadas por meio de índices de segregação e decomposições salariais com base na PNAD 2015 para uma amostra de pessoas com ensino superior completo. Os resultados apontam menor desigualdade entre os mais jovens, mas ainda deixam dúvidas se são efeitos de idade ou coorte. Para isso, uma segunda análise compara os índices de segregação da coorte entre 26 e 35 anos em 2015 com o mesmo intervalo nos anos de 1995 e 2005, com o intuito de separar os efeitos de coorte e de idade. Ao final, indica-se que a idade é mais associada ao aumento da desigualdade do que a coorte, embora entre os mais jovens haja menor segregação por raça.

Palavras-chave: Segregação ocupacional. Ocupações. Gênero. Raça. Desigualdade.

\footnotetext{
* Instituto de Pesquisa Econômica Aplicada (Ipea), Rio de Janeiro-RJ, Brasil (leosilveira.soc@gmail.com; https://orcid. org/0000-0002-9083-3123).

** Instituto de Estudos Políticos e Sociais, Universidade Estadual do Rio de janeiro (lesp/UERJ), Rio de Janeiro-RJ, Brasil (leaosnatalia@gmail.com; https://orcid.org/0000-0002-7925-3400).
} 


\section{Introdução}

0 mercado de trabalho brasileiro é caracterizado pela segmentação ocupacional dos homens negros, discriminação salarial contra as mulheres brancas e uma combinação dos dois efeitos sobre as mulheres negras (BRUSCHINI; LOMBARDI, 1999; SILVA, 1999; SOARES, 2000; OLIVEIRA; RIOS-NETO, 2004; 2006; SANTOS, 2005; 2008). Nesse contexto, comum às décadas de 1980 e 1990, foram inseridas algumas mudanças políticas e econômicas, como universalização do ensino fundamental, expansão do ensino superior, contínuo aumento da participação feminina desde os anos 1970, além da queda da contribuição da indústria de transformação a partir da década de 1980 (KON, 2006; PRATES, 2018; SILVEIRA; LEÃO, 2020).

Com base em tais considerações, o presente artigo investiga se essas mudanças foram suficientes para alterar o quadro de desigualdades por gênero e raça no mercado de trabalho brasileiro. A análise é realizada para uma amostra de pessoas com ensino superior completo em 2015, dividida em três grupos decenais entre 26 e 55 anos de idade. Em resultados prévios, demonstra-se que, ao se comparar a estrutura ocupacional brasileira entre 1986 e 2015, há uma redução da segregação e do diferencial de renda por gênero e raça (SILVEIRA; LEÃO, 2020). Porém, também são observadas heterogeneidades por região e por níveis escolares. Nas regiões Sul e Sudeste, a queda dos níveis de segregação é mais acentuada, ao passo que entre a população com ensino superior completo o diferencial salarial entre homens brancos e mulheres negras é maior do que no restante da população.

Isso, por um lado, foi acarretado pela diversificação da força de trabalho em termos de origem socioeconômica, gênero e raça. Por outro lado, a conversão desse cenário em redução de desigualdade é errática, sendo que os mecanismos de diferenciação se atualizam e cada coorte perpassa por diferentes composições e condições macroeconômicas (KORENMAN; NEUMARK, 1997). Nesse sentido, as análises temporais permitem uma visão comparativa das transformações ocorridas ao longo do tempo e auxiliam a responder: essas variações são sensíveis e equivalentes por idade? A desigualdade enfrentada pelos jovens é similar àquela sofrida pelos mais velhos, em termos de segregação ocupacional e diferencial salarial?

Para investigar tais diferenças etárias, realizam-se duas análises complementares com base em dados da PNAD para trabalhadores entre 26 e 55 anos com ensino superior completo. A primeira centra-se na análise de três índices de segregação e dois índices para decomposição dos diferenciais de renda (DEL Río; ALONSO-VILLAR, 2015). Essas análises são feitas de maneira comparada para os grupos de 26 a 35, 36 a 45 e 46 a 55 anos de idade em 2015. Os resultados demonstram que, de fato, os mais jovens apresentam um retrato menos desigual em termos ocupacionais e salariais.

Contudo, um segundo questionamento permanece: tal diferença deve-se a efeitos de idade ou de coorte? Para responder a essa indagação, comparam-se os índices de segregação da coorte nascida entre 1960 e 1969 nos anos de 1995 e 2005 - quando tinham de 
26 a 35 e 36 a 45 anos de idade -, buscando verificar se a segregação tende a ser menor entre os mais jovens ou se esta coorte possui aspectos que já indicavam maior segregação em décadas anteriores.

0 artigo conclui que a segregação por gênero e raça entre pessoas com ensino superior completo no Brasil é menor entre os mais jovens. Os índices de segregação e a desigualdade salarial são menores no grupo de 26 a 35 anos do que no de 46 a 55 anos. Com relação à segunda análise, demonstra-se, por um lado, que a coorte de nascidos entre 1960 e 1969 era menos segregada em 1995 do que em 2015, indicando que o mercado de trabalho tem dinâmicas que aumentam a segregação e a desigualdade ao longo das trajetórias ocupacionais, embora, por outro lado, seja verificada a diminuição da segregação por raça na coorte de nascidos entre 1980 e 1989.0 artigo se divide em cinco partes, além dessa introdução. Na próxima seção, abordam-se as hipóteses que explicam como as mudanças estruturais podem diminuir as desigualdades adscritas. Posteriormente, são apresentados o desenho de pesquisa e a construção dos índices analisados, seguidos pelos resultados. Por fim, são discutidos os resultados para as duas análises temporais acerca do contexto brasileiro.

\section{Efeitos de idade, período e coorte por gênero e raça no mercado de trabalho}

A segregação ocupacional reflete a associação entre a inserção em postos de trabalho e características, sejam estas produtivas (experiência ou escolaridade, por exemplo), sejam adscritas (gênero, raça, nacionalidade, origem socioeconômica, entre outras). Para além da inserção, outro viés que a estrutura ocupacional intermedia é o retorno salarial que, nas palavras de Carvalhaes (2015), relaciona-se à distribuição dos recursos e bens gerados no processo produtivo. Logo, a concentração sistemática de algum grupo em ocupações com maiores salários médios conduz a um aumento na desigualdade em relação aos demais (SOARES, 2000; SANTOS, 2008; BOTASSIO; VAZ, 2020).

Nesse ciclo, o gênero tem um papel preponderante para a segmentação da estrutura ocupacional. Primeiramente, a teoria econômica neoclássica defende que os retornos salariais se dão pela contribuição marginal dada por cada indivíduo à função produção. Isto é, homens e mulheres teriam atributos produtivos e preferências distintas entre si, sendo que as diferenças salariais seriam consequências de escolhas desses grupos (BECKER, 1957). Por outro lado, hipóteses relacionadas à socialização e à discriminação confrontam tal perspectiva. No primeiro caso, mulheres e homens são socializados em papéis e em meio a instituições que direcionam suas escolhas no que diz respeito à divisão sexual do trabalho pago e não pago (HIRATA; KERGOAT, 2007). Como pontua Santos (2008), ao se observar a existência de discriminação salarial, abre-se espaço para hipóteses concorrentes à neoclássica. A discriminação estatística considera que, uma vez que o empregador não possui informações suficientes e completas sobre o empregado, ele utiliza o gênero (entre outras características atribuídas - raça, nacionalidade, etc.) como informação substituta de produtividade e definição salarial. 
Além disso, os padrões de segregação descrevem uma sobrerrepresentação de mulheres em ocupações chamadas de "tipicamente femininas". Estas são caracterizadas por categorias de profissionais assalariados (white-collar) e no setor de cuidados e reprodução da vida, que apresentaram considerável crescimento nas economias pós-industriais (BRUSCHINI, 2000; GUIMARÃES et al., 2011; BOTASSIO; VAZ, 2020). Ou seja, crenças culturais levam a uma expectativa de que ocupações como professoras de educação básica, enfermeiras, cuidadoras e trabalhadoras de serviços administrativos sejam mais bem executadas por mulheres do que por homens, embora não haja nenhuma justificativa prévia para essa diferenciação. ${ }^{1}$ As consequências da segregação, dos papéis de gênero e da discriminação estatística são salários mais baixos das mulheres, mesmo que se equivalham em posições e qualificações (SOARES, 2000; SANTOS, 2008).

Embora o gênero seja o marcador não produtivo mais relevante para a segregação ocupacional, a raça também se mostra determinante e persistente (SILVEIRA; LEÃO, 2020). Em estudo anterior, observamos um "elitismo" da discriminação (SILVEIRA; LEÃO, 2020), em que trabalhadores negros, ao atingirem posições hierarquicamente mais elevadas, sofrem mais com a discriminação (SOARES, 2000; CAMPANTE et al., 2004; BAILEY et al., 2013), chamado também de interação estrutural por Santos (2005).

A coextensão entre raça e gênero se expressa nos planos discursivo, político e socioeconômico (KERGOAT, 2010). No mercado de trabalho, Soares (2000) decompôs a desigualdade salarial entre quatro grupos de gênero e raça - homens negros, homens brancos, mulheres negras e mulheres brancas - e demonstrou que ela ocorre em função de três mecanismos: formação, inserção e discriminação. Em relação aos homens brancos, os homens negros têm desvantagem por formação e inserção, já que eles possuem menor escolaridade média e estão inseridos em ocupações de menor prestígio, principalmente manuais. Para as mulheres brancas, pesam a inserção e a discriminação, dado que elas se encontram em ocupações intermediárias e, mesmo tendo maior escolaridade e estando nos mesmos cargos, recebem menos. Por fim, as mulheres negras sofrem com desvantagens combinadas entre os três mecanismos. Em valores percentuais, em 1987, para os homens negros, $11 \%$ da sua diferença em relação aos homens brancos devia-se à discriminação, sendo que esse valor era de $37 \%$ para as mulheres brancas e $48 \%$ para as negras. Em 1998 , esses percentuais passaram para $15 \%, 29 \%$ e $39 \%$, respectivamente (SOARES, 2000).

Em uma análise mais recente, Botassio e Vaz (2020) demonstram que, entre 2004 e 2015, a segregação ocupacional por gênero no Brasil se tornou mais forte. Segundo os autores, a queda na segregação ocasionada pelo aumento proporcional das mulheres tem atingido um esgotamento. Ao fazer um recorte por coorte, inclusive, eles apontam que 0 nível de segregação, por um lado, é maior entre os mais velhos (coorte de 1946 a 1964) do

\footnotetext{
${ }^{1}$ England (2008) apresenta sínteses de pesquisas sociológicas e econômicas sobre os efeitos da concentração de mulheres em determinadas ocupações. Resultados apontam que certas categorias têm salários menores, sendo que a única diferença está no percentual de mulheres que as ocupam. Além disso, mesmo nessas ocupações, as mulheres tendem a ter menores salários do que os homens que as ocupam.
} 
que entre os mais jovens (1981 a 1996), mas, por outro, tem se tornado maior nesse último grupo em comparação ao primeiro (BOTASSIO; VAZ, 2020). Vale pontuar, no entanto, que o acréscimo da variável racial nesta análise pode contribuir para a compreensão dessas tendências da segregação por gênero.

Nesse sentido, questiona-se se a estrutura ocupacional brasileira se alterou nas últimas décadas. Como apresentado por Silveira e Leão (2020), entre 1986 e 2015, o mercado de trabalho por raça e gênero se tornou ligeiramente menos segregado no país, sendo menor o diferencial de renda atribuído à segregação para mulheres negras e brancas e homens negros. Em um recorte mais elitizado, de trabalhadores com ensino superior, porém, o termo de discriminação contra as mulheres brancas diminuiu ligeiramente, enquanto para as negras e os negros esse percentual até aumentou.

As análises temporais, portanto, demonstram que os índices de segregação e discriminação por gênero e raça apresentam melhoras lentas e erráticas. Isso contraria, de certo modo, as expectativas da chamada teoria da modernização, que preconizava que 0 desenvolvimento da ordem competitiva tornaria as desigualdades de origem menos aceitáveis, uma vez que ela é contraditória à ideologia meritocrática (PARSONS, 1951). Nesse sentido, indaga-se se o cenário observado da segregação e da discriminação salarial em 2015 era constante entre os recortes etários, uma vez que, como demonstrado por Oliveira e Rios-Neto (2006), variações temporais no mercado de trabalho ocorrem por meio dos grupos de idade, período ou coorte.

Para Oliveira e Rios-Neto (2006), mudanças decorrentes de idade devem-se a fatores cumulativos ao longo do ciclo de vida, como experiência e treinamento, ou seja, relacionados ao capital humano. Na Suécia, Bronson e Thoursie (2017) sustentam que a idade tem um efeito positivo sobre as desigualdades salariais por gênero, ao demonstrarem que, após sequências de promoções e mudanças de empregos, homens passam a receber entre $73 \%$ e $83 \%$ a mais do que as mulheres.

Por sua vez, os efeitos de períodos são externos e em geral correspondem ao ambiente econômico, sinalizado por crises ou fases de crescimento contínuo. Por fim, os efeitos de coorte são representados por mudanças que afetam todo um grupo de nascidos em determinado intervalo e que são expostos a condições similares, como a massificação ou a alteração na qualidade de ensino, por exemplo (OLIVEIRA; RIOS-NETO, 2006). Em outras palavras, o recorte por grupos etários pretende analisar se os mais jovens experimentam menor desigualdade do que os mais velhos.

No Brasil, Barbosa (2016) analisa os efeitos de idade, período e coorte na desigualdade de renda para homens e mulheres separadamente. Entre 1981 e 2013, o autor identifica que os homens experimentaram um processo de diminuição da desigualdade, principalmente entre as coortes mais jovens, que coincide com o momento de expansão do ensino básico no país. Entre as mulheres, o processo de redução das desigualdades se deu em períodos específicos, atingindo todas as idades e coortes, principalmente a partir de 1993-1994 (BARBOSA, 2016). 
Costa Ribeiro (2016) também separa homens e mulheres para estimar os efeitos de idade, período e coorte na associação entre ter diploma superior e alcançar o topo da hierarquia ocupacional. 0 autor observa que, tanto para os homens quanto para mulheres, os principais efeitos estão no período em vez da coorte. Ele demonstra que, a partir de meados da década de 1990, ocorreu uma "inflação de credenciais", no sentido de que, mesmo com as crises econômicas, o percentual de diplomados cresceu, mas não foi acompanhado pela demanda por esses profissionais. Nesse sentido, a associação entre o diploma e as mais altas ocupações diminuiu para todos os grupos em períodos mais recentes, independentemente da coorte de nascimento, idade e gênero (COSTA RIBEIRO, 2016).

Em termos raciais, Reis e Crespo (2015) colocam a discriminação entre homens negros e brancos no Brasil sob análise de idade, período e coorte. Os autores destacam que a discriminação é mais forte quando os trabalhadores estão no início de sua trajetória profissional, de maneira que, ao adquirirem mais experiência, os trabalhadores negros passam a sofrer menos com a discriminação. Por coorte, porém, aqueles nascidos após a década de 1970 sofrem menos com a discriminação do que os nascidos anteriormente aos anos 1940.

Quando analisamos a segregação por raça e gênero nas últimas três décadas, verificamos uma queda na segregação e na discriminação, embora cercada de heterogeneidades escolares e regionais (SILVEIRA; LEÃO, 2020). Além disso, apesar desta redução, questionou-se quais aspectos estariam associados a tal mudança no tempo. Nesse sentido, a análise que se segue tem como objetivo principal investigar a segregação ocupacional por grupos etários. Analisando dados da PNAD 2015, pode-se dizer que a segregação é maior ou menor entre os mais jovens? Com base nos resultados, espera-se discutir se há evidências que demonstrem maior equalização em termos ocupacionais nas coortes mais jovens. Contudo, um segundo questionamento é se essa diferença deve-se a efeitos de idade ou de coorte. Para isso, comparam-se os índices de segregação da coorte nascida entre 1960 e 1969 em 1995 e 2005 - quando tinham entre 26 e 35 e 36 e 45 anos de idade -, buscando responder se os resultados encontrados para 2015 dizem respeito a um efeito de coorte ou de idade.

\section{Dados e metodologia}

Para testar as diferenças etárias, a estratégia de análise se baseia no uso de três índices de segregação e dois de decomposição salarial desenvolvidos por Del Río e Alonso-Villar (2015). Estes permitem uma descrição da segregação e da desigualdade salarial, incluindo todos os grupos de gênero e raça de forma conjunta, diferentemente de índices usualmente utilizados, como o índice de dissimilaridade, ou de modelos de idade-período-coorte (IPC). Estes últimos, em especial, são empregados para medir variações temporais, comumente usando variáveis binárias, em geral, o gênero. No caso do presente artigo, optou-se pelos índices dada a possibilidade de comparar diferentes subamostras no mesmo ano. ${ }^{2}$

\footnotetext{
$\overline{2}$ Para tais análises, ver Rios-Neto e Oliveira (1999), Oliveira e Rios-Neto (2004, 2006), Costa Ribeiro (2016) e Barbosa (2016).
} 
Com base na PNAD 2015, o desenho da análise foi feito para três grupos etários no Brasil de pessoas com ensino superior completo, sendo o grupo 1 de 26 a 35 anos, o grupo 2 de 36 a 45 anos e o último de 46 a 55 anos. A opção por grupos decenais a partir dos 26 anos e com o mesmo nível de escolaridade se deu pelo fato de a análise usar dados observados, e não preditos, sendo uma maneira de assegurar maior homogeneidade. Já a PNAD 2015 é a edição mais recente da pesquisa em formato anual, permitindo comparabilidade com anos anteriores, além de ser a mesma edição utilizada por Silveira e Leão (2020), que servem de contraponto aos atuais resultados.

Tal comparação com anos anteriores será feita para responder ao objetivo secundário de analisar se as diferenças na segregação ocupacional por grupos etários devem-se a especificidades por coortes. Para isso, analisam-se os índices de segregação da coorte nascida entre 1960 e 1969 - ou seja, o mesmo grupo 3 em 2015, porém em idades mais jovens - quando seus indivíduos tinham a mesma idade das coortes 1 e 2 em décadas anteriores, nas PNAD 1995 e 2005 . Assim, pode-se mensurar se essa coorte tem padrões menos igualitários em comparação àquelas mais jovens, ou se em idades mais avançadas os índices de segregação tendem a aumentar, deflagrando um efeito etário. A seguir, são apresentados o banco de dados e a amostra analítica, as variáveis que balizam a análise e a descrição dos índices de segregação ocupacional e os diferenciais salariais.

\section{Banco de dados e amostra analítica}

De acordo com o desenho proposto para analisar as duas perguntas de pesquisa variação por idade e variação da coorte mais velha ao longo de três décadas -, a Pesquisa Nacional por Amostra de Domicílios (PNAD) permite que se tenha uma cobertura representativa tanto dos três grupos em 2015 quanto da coorte de nascidos entre 1960 e 1969, a ser analisada também em 1995 e 2005. Isto é, utilizou-se a última PNAD em sua versão anual, que pode ser comparada com as edições em décadas anteriores, o que não seria possível com as coletas mais recentes da PNAD Contínua.

A PNAD é representativa para o Brasil rural e urbano desde 2003, sendo que, anteriormente, isso não era observado para a população rural da região Norte (BARBOSA, 2014). Entretanto, uma vez que o segundo objetivo deste trabalho é comparar a coorte de nascidos entre 1960 e 1969 em décadas anteriores, optou-se por manter o desenho amostral anterior a 2003, sem considerar a população rural da região Norte.

As amostras analíticas, então, foram feitas considerando os dois objetivos. Para o primeiro, têm-se trabalhadores entre 26 e 55 anos que tinham alguma ocupação em 2015. Há também uma restrição por escolaridade, apenas para aqueles com ensino superior completo, com o intuito de diminuir a variação do perfil e da qualificação dos trabalhadores, dado que não se trata de um modelo multivariado. ${ }^{3}$ Já para a segunda pergunta, consideram-se os trabalhadores com ensino superior que nasceram entre 1960 e 1969 e tinham alguma

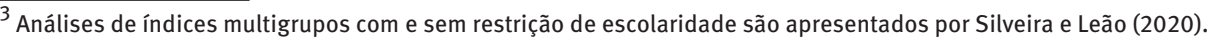


ocupação em 1995 e 2005, para compará-los aos resultados de 2015. Com isso, têm-se uma análise transversal em 2015 e uma análise longitudinal em três diferentes décadas.

\section{Variáveis}

Os índices são apresentados na seção subsequente e trazem medidas globais e locais de segregação ocupacional, bem como de diferenciais de renda por raça e gênero. Para isso, então, são necessárias as variáveis de ocupação, gênero, raça e rendimento do trabalho principal.

Para mensurar as ocupações, utilizou-se a Classificação Brasileira de Ocupações (CBO). Em geral, as classificações ocupacionais traduzem e agregam características relacionadas ao trabalho realizado pelos indivíduos. As variáveis de gênero e raça buscam encontrar regularidades nos seus padrões de ocupações e diferenciais de rendimentos. Por isso, o gênero está dividido de maneira binária - mulheres e homens -, considerando as discussões sobre o impacto da construção social de gênero nas formas de socialização que acabam levando a concentrações em termos ocupacionais. Além disso, vale destacar que a PNAD não mensura outras categorias de gênero. Por raça, foram excluídas as categorias indígena e amarela, dividindo a amostra analítica entre negros (pretos e pardos) e brancos, sendo esses os principais marcadores raciais para segregação ocupacional. Ademais, vale acrescentar que ambas as variáveis são analisadas de maneira combinada, tendo, portanto, resultados para mulheres negras, mulheres brancas, homens negros e homens brancos, considerando-se que cada um desses grupos possui especificidades que merecem ser tratadas de forma separada.

A última variável é a renda, que se restringiu ao rendimento do trabalho principal dividido pelo número de horas trabalhadas por semana multiplicado por 44 , como maneira de padronizar a variável mesmo dentre aqueles que não trabalham por período completo. Vale ressaltar que, em 2015, 33\% dos trabalhadores tinham jornada de 40 horas e 17\% de 44 horas, configurando metade da amostra total.

\section{Índice multigrupo}

A segregação ocupacional e o diferencial de rendimentos são medidos por meio do local segregation measures, desenvolvido por Del Río e Alonso-Villar (2015). Trata-se de um conjunto de cinco índices com objetivos distintos. Destes, três dizem respeito à segregação dos grupos de gênero e raça dentro da estrutura ocupacional e dois relacionam-se à renda média desses grupos. Todos os índices são sintetizados e têm suas notações no Quadro 1.

0 índice 1 , chamado de local segregation, reflete a concentração ou dispersão de cada um dos grupos de gênero/raça definidos. 0 índice 2 é uma medida global da segregação $(M)$ e possibilita comparar a estrutura em diferentes pontos do tempo. Nesse caso, será comparada a estrutura ocupacional entre aqueles nascidos nas coortes 1, 2 e 3, além da coorte de nascidos entre 1960 e 1969 em diferentes momentos do tempo. 0 terceiro índice é a contribuição do grupo para o índice global e sintetiza quanto da segregação geral pode ser atribuído a cada um dos grupos analisados. Os dois últimos índices, 4 e 5, são 
complementares na análise da desigualdade de renda por se tratarem de uma decomposição. 0 índice 4 mensura o quanto da renda média do grupo de gênero/raça deve-se ao fato de ele estar em ocupações mais ou menos valorizadas. Isto é, homens brancos, em geral, se concentram em ocupações que têm retornos salariais mais altos, sendo esse índice positivo, enquanto para mulheres negras ele será negativo, por estarem em ocupações com menor remuneração média. 0 índice 5 mede o quanto cada grupo ganha ou perde devido à discriminação salarial. Esse é, grosso modo, a média das diferenças do salário médio de cada grupo de raça/gênero em comparação às médias das ocupações.

QUADRO 1

Fórmulas e interpretações dos índices de local segregation

\begin{tabular}{|c|c|c|c|}
\hline $\mathbf{N}$ & Fórmulas & Símbolos & Interpretação \\
\hline 1 & $\Phi_{1}^{g}(c ; t)=\sum_{j} \frac{c_{j}^{g}}{C^{g}} \ln \left(\frac{\frac{c_{j}^{g}}{C^{g}}}{\frac{t_{j}}{T}}\right)$ & $\begin{array}{l}\text { g: grupo de raça/gênero } \\
\text { específico } \\
t_{j} \text { : total de indivíduos em } \\
\text { cada ocupação } j ; \\
C_{j}^{g} \text { : quantidade de } \\
\text { indivíduos de cada grupo } g \\
\text { em cada ocupação } j ; \\
C^{g} \text { : total de indivíduos do } \\
\text { grupo } g \text { na amostra; } \\
T \text { : total de observações na } \\
\text { amostra. }\end{array}$ & $\begin{array}{l}\text { O índice sintetiza o quanto cada grupo } \\
\text { de raça/gênero (g) está segregado } \\
\text { naquela sociedade. Quanto mais baixa } \\
\text { for a representatividade de um grupo } \\
\text { em cada ocupação }(t) \text {, maior será a } \\
\text { contribuição para o índice. Se, por outro } \\
\text { lado, a representatividade nas ocupações } \\
\text { for sempre equivalente à sua parcela na } \\
\text { população geral, o índice será zero. Quanto } \\
\text { mais os grupos de raça e gênero forem } \\
\text { segregados em determinadas ocupações, } \\
\text { maior o seu valor (varia entre } 0 \text { e o máximo } \\
\text { valor de } \ln (T) \text { ). }\end{array}$ \\
\hline 2 & $M=\sum_{g} \frac{C^{g}}{T} \Phi_{1}^{g}$ & Ф: resultado do índice 1. & $\begin{array}{l}\text { O índice } M \text { reflete toda a estrutura } \\
\text { ocupacional. Dados os resultados para } \\
\text { todos os grupos g no índice } 1 \text {, o índice } \\
M \text { aponta que, quanto maior o seu } \\
\text { valor, maior será a segregação naquela } \\
\text { população (variando entre } 0 \text { e 1). Além } \\
\text { disso, o índice é decomposto por raça e } \\
\text { gênero, indicando o peso de cada variável } \\
\text { para a segregação da população. }\end{array}$ \\
\hline 3 & $\begin{array}{l}\text { Contribuição do grupo } \\
g=\frac{\frac{C^{g}}{T} \Phi_{1}^{g}}{M}\end{array}$ & M: resultado do índice 2. & $\begin{array}{l}\text { O índice de contribuição aponta a } \\
\text { influência de cada um dos grupos para } \\
\text { o índice global } M(2) \text {. Logo, se o valor de } \\
\text { um grupo for 0,30, quer dizer que 30\% do } \\
\text { índice global deve-se à sua distribuição na } \\
\text { estrutura. }\end{array}$ \\
\hline 4 & $r=\sum_{j}\left(\frac{C_{j}^{g}}{C^{g}}-\frac{t_{j}}{T}\right) \frac{w_{j}}{W^{\prime}}$ & $\begin{array}{l}w_{j} \text { : rendimento médio do } \\
\text { trabalho principal para cada } \\
\text { ocupação; } \\
\text { w': rendimento médio global. }\end{array}$ & $\begin{array}{l}\text { O índice } 4 \text { também é dado por grupo e } \\
\text { reflete o quanto do salário médio de cada } \\
\text { um é influenciado pela segregação. Logo, } \\
\text { se um grupo estiver em ocupações que } \\
\text { pagam salários maiores, seu índice será } \\
\text { positivo e maior; não há limites de variação, } \\
\text { podendo ser positivo ou negativo, e é lido } \\
\text { em termos de proporção ou percentual. }\end{array}$ \\
\hline 5 & $\Delta=\left\ulcorner\sum_{j} c_{j}^{g}\left(W_{j}^{g}-W_{j}\right)\right\rceil \frac{1}{C^{g} W^{\prime}}$ & $\begin{array}{l}W_{\dot{j}}^{g}: \text { renda média do grupo } \\
g \text { ha ocupação } j .\end{array}$ & $\begin{array}{l}\text { O valor de delta também é dado para cada } \\
\text { um dos grupos, refletindo a soma das } \\
\text { diferenças entre a renda média de cada } \\
\text { grupo g em cada ocupação j e a média } \\
\text { de renda da ocupação j. Trata-se de uma } \\
\text { medida de discriminação salarial, já que } \\
\text { reflete as diferenças de rendimento por } \\
\text { grupo de indivíduos que preenchem as } \\
\text { mesmas ocupações. Também é lido em } \\
\text { termos percentuais. }\end{array}$ \\
\hline
\end{tabular}

Fonte: Del Río e Alonso-Villar (2015). 


\section{Resultados}

As coortes de idade podem ser distintamente impactadas por situações estruturais, como índices de desemprego, crises que afetam toda uma geração e/ou taxas de escolaridade variável (KORENMAN; NEUMARK, 1997). Antes de se analisarem os índices de segregação, observa-se que na PNAD 2015 - sem fazer as restrições para a amostra analítica -, nenhum dos três grupos etários apresenta variações significativas no percentual de participação na população economicamente ativa (PEA) - 90,6\% no grupo 1, 93,7\% no grupo 2 e 95,2 no grupo 3 -, ou no percentual de indivíduos com ensino superior completo - 16,3\%, 14,5\% e 13,7\%, respectivamente. Assim, embora sejam medidas simples, não há sinais de variações intercoortes que possam ser atribuídas a aspectos macrossociais.

De agora em diante, as análises são restritas à subamostra analítica, apenas para os indivíduos brancos, pretos e pardos, com ensino superior completo, com alguma ocupação em 2015 e em idades entre 26 e 55 anos. Na Tabela 1, observam-se a composição por raça e gênero, a razão salarial e a média e mediana do salário de cada coorte.

TABELA 1

Estatísticas descritivas da amostra analítica

Brasil - 2015

\begin{tabular}{|c|c|c|c|c|c|c|}
\hline \multirow[b]{2}{*}{ Categorias } & \multicolumn{2}{|c|}{ Grupo 1 (26 a 35 anos) } & \multicolumn{2}{|c|}{ Grupo 2 (36 a 45 anos) } & \multicolumn{2}{|c|}{ Grupo 3 ( 46 a 55 anos) } \\
\hline & $\%$ & $\begin{array}{c}\text { Razão } \\
\text { salarial (1) }\end{array}$ & $\%$ & $\begin{array}{c}\text { Razão } \\
\text { salarial (1) }\end{array}$ & $\%$ & $\begin{array}{c}\text { Razão } \\
\text { salarial (1) }\end{array}$ \\
\hline Homens brancos & 27,8 & (ref.) & 27,4 & (ref.) & 27,8 & (ref.) \\
\hline Homens negros & 15,2 & 76,0 & 16,1 & 69,0 & 15,9 & 57,0 \\
\hline Mulheres brancas & 36,2 & 62,0 & 33,9 & 78,0 & 36,0 & 59,0 \\
\hline Mulheres negras & 20,8 & 51,0 & 22,6 & 48,0 & 20,3 & 44,0 \\
\hline Salário médio (em R\$) (2) & \multicolumn{2}{|c|}{$6.818,47$} & \multicolumn{2}{|c|}{$7.759,42$} & \multicolumn{2}{|c|}{$9.065,24$} \\
\hline Mediana (em R\$) (2) & \multicolumn{2}{|c|}{$2.970,00$} & \multicolumn{2}{|c|}{$3.520,00$} & \multicolumn{2}{|c|}{$3.850,00$} \\
\hline Observações & \multicolumn{2}{|c|}{7.296} & \multicolumn{2}{|c|}{6.388} & \multicolumn{2}{|c|}{4.916} \\
\hline Ocupações & \multicolumn{2}{|r|}{289} & \multicolumn{2}{|c|}{285} & \multicolumn{2}{|r|}{270} \\
\hline
\end{tabular}

Fonte: IBGE. PNAD 2015.

(1) Razão salarial multiplicada por 100. Salário-horário dos homens brancos como referência.

(2) Em valores correntes, não corrigidos.

No que diz respeito aos salários recebidos por cada grupo etário, pode-se dizer que, em primeiro lugar, as disparidades são menores entre os mais jovens e o salário médio é maior entre os mais velhos. A diferença entre a razão salarial entre homens brancos e demais grupos é menor na coorte 1, em que as mulheres negras recebem $51 \%$ do salário dos homens brancos, sendo que na coorte 3 esse percentual é de $44 \%$. Os homens negros de 26 a 35 anos recebem $76 \%$ do salário de seus congêneres brancos, e $57 \%$ quando têm entre 46 e 55 anos. Contudo, vale destacar que as mulheres brancas na coorte 2 (36 a 45 
anos) se encontram em melhor condição salarial do que aquelas da coorte 1, em relação aos homens de suas respectivas faixas etárias.

A composição dos grandes grupos ocupacionais da Classificação Brasileira de Ocuapções (CBO) ${ }^{4}$ permite observar como determinadas áreas são segmentadas por gênero e raça (Gráfico 1). Para sua análise, tem-se um olhar tanto "vertical”, entre as ocupações, quanto "horizontal", ao longo das coortes. Verticalmente, os grupos de gênero e raça se distribuem de maneira desigual, sendo que, por exemplo, os homens predominam nas categorias 0 (Forças Armadas e militares), 6 (trabalhadores agropecuários, florestais e da pesca), 7 (trabalhadores da produção de bens e serviços industriais) e 9 (trabalhadores em serviços de reparação e manutenção). Horizontalmente, pontuam-se diferenças nas ocupações dos grupos 6, 8 (trabalhadores da produção de bens e serviços Industriais) e 9. No primeiro, os homens brancos representavam $60 \%$ do seu total entre trabalhadores de 46 a 55 anos, enquanto entre os outros dois intervalos esse percentual correspondia a $40 \%$. No grupo 8 , os homens negros e brancos passaram de $53 \%$ entre os mais velhos para 33\% entre os mais jovens, revelando uma maior participação de mulheres.

GRÁFICO 1

Composição dos grandes grupos ocupacionais (1), por recorte etário, segundo sexo e cor/raça Brasil - 2015
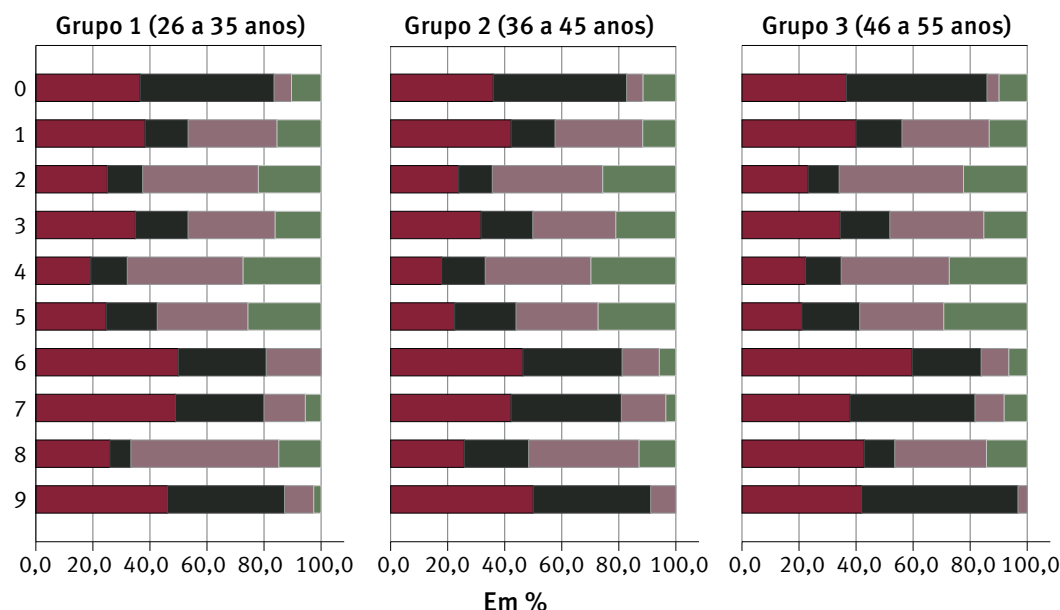

Homens brancos

Mulheres brancas

Homens negros

Mulheres negras

Fonte: IBGE. PNAD 2015

(1) Grandes grupos ocupacionais da Classificação Brasileira de Ocupações: 0 - Membros das Forças Armadas, policiais e bombeiros militares; 1 - Membros superiores do poder público, dirigentes de organizações de interesse público e gerentes; 2 - Profissionais das ciências e das artes; 3 - Técnicos de nível médio; 4 - Trabalhadores de serviços administrativos; 5 Trabalhadores dos serviços, vendedores do comércio em lojas e mercados; 6 - Trabalhadores agropecuários, florestais e da pesca; 7 - Trabalhadores da produção de bens e serviços industriais; 8 - Trabalhadores da produção de bens e serviços industriais; 9 - Trabalhadores em serviços de reparação e manutenção.

\footnotetext{
${ }^{4}$ Como observado na Tabela 1, em cada intervalo etário há, pelo menos, 270 ocupações. Assim, com o intuito de melhorar a visualização, utilizam-se apenas os grandes grupos da CBO para representar a segmentação ocupacional.
} 
Os demais grupos não apresentam variações significativas entre grupos etários. Essa estabilidade reflete a consistência da segmentação ocupacional. Em conjunto, as estatísticas descritivas apresentadas na Tabela 1 e no Gráfico 1 demonstram um cenário de diferenciações por gênero e raça, retratando pequenas variações por grupos etários, sem que se altere substantivamente o padrão de desigualdades. Quando existem, as variações são favoráveis ao grupo jovem, com grupos ocupacionais mais diversos e menores disparidades salariais.

No Gráfico 2, apresenta-se o índice 1, o mais central para a medição da segregação, com valores agregados que medem a concentração/dispersão dos grupos de gênero e raça nas ocupações. De maneira geral, os valores mais baixos apontam uma melhor distribuição dos mais jovens. As mulheres brancas têm índices mais baixos do que os demais - ou seja, elas estão mais distribuídas entre as ocupações. Os homens negros apresentam segregação mais alta, especialmente na faixa entre 46 e 55 anos, refletindo, por exemplo, que eles têm maior concentração nos grandes grupos 0, 6, 7 e 9. Por fim, homens brancos e mulheres negras, que são grupos historicamente segregados e circunscritos a determinadas ocupações, também têm menores índices entre trabalhadores mais jovens. Em suma, o que se observa no Gráfico 2 é a manutenção do padrão mais baixo de segregação entre as mulheres brancas, além de um padrão mais baixo em todos os grupos de cor/raça e gênero nos grupos mais jovens.

\section{GRÁFICO 2}

Índice local segregation, por recorte etário, segundo sexo (índice 1) Brasil - 2015

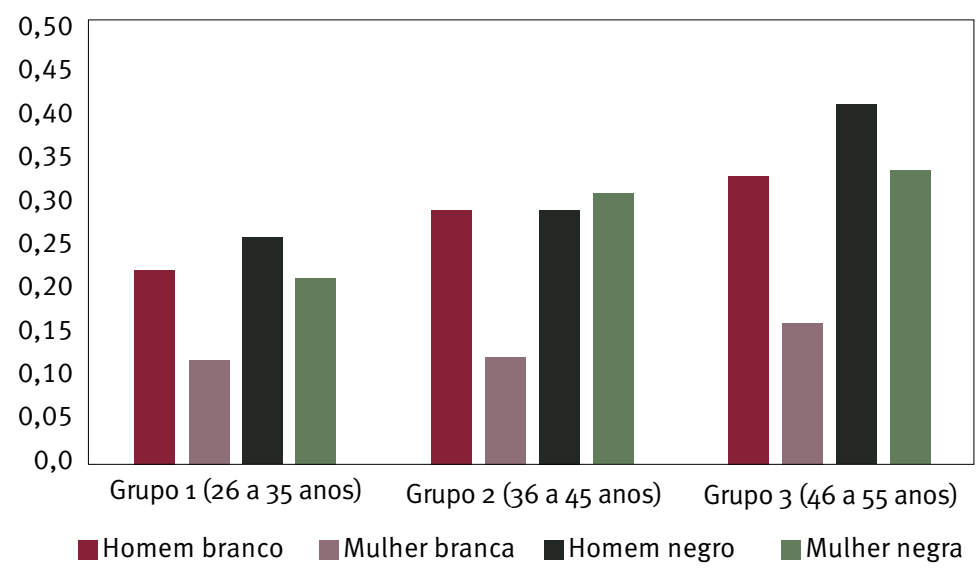

Fonte: IBGE. PNAD 2015

O índice 2, chamado por Del Río e Alonso-Villar (2015) de $M$, é uma medida global que pode ser decomposta na contribuição do gênero e da raça para o índice total. Os valores, que podem ser consultados na Tabela do Anexo, indicam que a segregação global é maior entre o grupo 3, mais velho, cujo índice é de 0,2827, contra 0,2386 para o grupo de 36 a 45 anos e 0,1889 para o de 26 a 35 anos. Os resultados também mostram que o gênero 
tem uma contribuição consideravelmente maior do que a cor/raça: entre os mais velhos, o gênero tem índice de 0,2011 e a cor/raça 0,0639, enquanto entre os mais jovens esses valores são de 0,1327 e 0,0393, respectivamente.

0 terceiro índice - contribuição do grupo $g$ - trata-se de um valor padronizado entre 0 e 1 (ou, leia-se, entre $0 \%$ e $100 \%$ ) e aponta qual a fatia de toda a segregação medida deve-se àquele grupo - em um cenário ideal, cada grupo teria índice igual a $25 \%$. Dentre os mais jovens (grupo 1), apenas os homens brancos superaram esse valor (33\%). Isso quer dizer que se trata de um grupo mais restrito a determinadas ocupações do que a outras. Já os demais grupos apresentaram índice de 23\%, para mulheres brancas e negras, cada, e $20 \%$ para homens negros. Os valores do grupo mais jovem são similares ao observado para as demais coortes. Entre as variações temporais, destaca-se o aumento no grupo 2 para mulheres negras, atingindo $29 \%$, sendo o único grupo que ultrapassou os $25 \%$, além dos homens brancos.

Estes três primeiros índices apresentados dizem respeito à distribuição de grupos de gênero e cor/raça nas ocupações. Como se observou, os grupos não são homogeneamente distribuídos entre as ocupações. Contudo, como isso afeta o rendimento dos indivíduos? 0 Gráfico 3 apresenta os dois últimos índices, que medem a decomposição da renda média dos grupos, tendo um termo referente à segregação e outro relacionado à discriminação.

GRÁFICO 3

Diferença salarial, segundo diferenças de composição ocupacional (índice 4) e discriminação (índice 5) Brasil - 2015

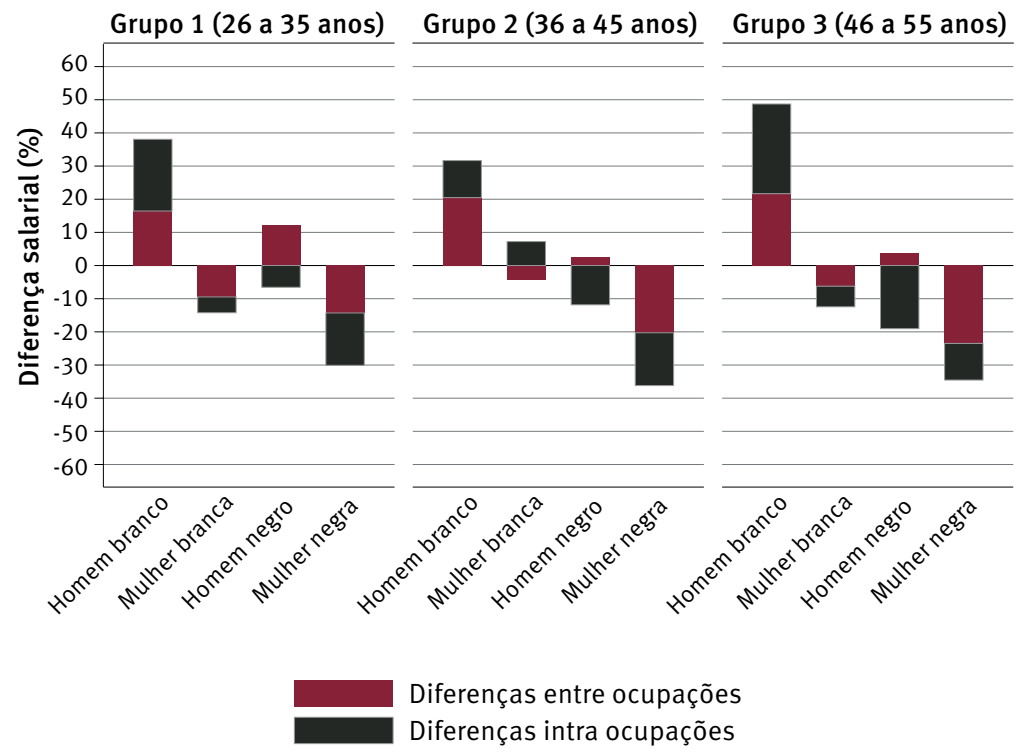

Fonte: IBGE. PNAD 2015. 
Ambos os índices apontam para uma vantagem consistente e constante dos homens brancos. Entre os mais jovens, o salário é quase $40 \%$ acima da média, sendo $16 \%$ referentes à segregação e $22 \%$ à discriminação. Isso significa que os homens brancos estão proporcionalmente sobrerrepresentados em ocupações de salários médios maiores e, em geral, recebem salários maiores, mesmo quando estão nas mesmas categorias. No grupo 2 , de 36 a 45 anos, essa vantagem é de $31 \%$, sendo o termo de discriminação de $11 \%$. Por último, na coorte mais velha, o diferencial atinge o ponto mais alto, sendo que os homens brancos recebem $49 \%$ a mais do que a média, com um termo de discriminação de $27 \%$. As mulheres brancas da coorte 1 , por sua vez, têm rendimento médio desvantajoso de $14 \%$ em relação à média. Esse percentual se torna positivo em 3\% na coorte intermediária e depois atinge um valor de $-12 \%$ na coorte mais velha.

0 grupo negro tem um padrão explícito de discriminação. Para os homens, o termo de discriminação se torna mais alto nas coortes mais velhas, sendo que, por outro lado, há vantagens salariais por estarem em ocupações mais bem posicionadas.

Porém, tanto para homens quanto para mulheres negros, em todos os intervalos etários, há desvantagens referentes à discriminação. As mulheres negras sofrem com os efeitos do sexismo e do racismo. No grupo etário menos desvantajoso, o grupo 1, elas recebem $30 \%$ a menos do que a média, sendo $16 \%$ devido à discriminação. No grupo 2 , essa desvantagem é de $-36 \%$, também com $16 \%$ referentes à discriminação, e no grupo 3 a desvantagem é de $-34 \%$, com $11 \%$ por discriminação.

Nascidos entre 1960 e 1969: efeitos de coorte ou expansão da desigualdade por idade?

A análise dos intervalos etários em 2015 demonstra que a segregação e os diferenciais de renda são maiores entre o grupo mais velho, de 46 a 55 anos. Contudo, ainda resta uma dúvida se o grupo nascido entre 1960 e 1969 sempre foi mais segregado ou se foi se tornando mais segregado com o tempo - isto é, a coorte mais velha possui um padrão de segregação mais forte do que as outras duas, ou a segregação ocupacional se tornou mais intensa com a idade? Para isso, analisou-se a variação dos índices de segregação da coorte de nascidos entre 1960 e 1969 nas PNADS de 1995 e 2005, quando estes indivíduos tinham de 26 a 35 e de 36 a 45 anos, respectivamente.

Para fins comparativos, analisaram-se os índices 1 e 2. Por meio do índice 1 (Gráficos 4 e 5), foram comparados os grupos quando tinham de 26 a 35 e de 36 a 45 anos. No primeiro caso, observa-se que os índices para homens e mulheres brancos são muito semelhantes, independentemente do ano. Já homens e mulheres negros entre 26 e 35 anos demonstram ser mais segregados em 1995 do que em 2015. Embora eles continuem sendo mais segregados do que seus congêneres brancos, o índice cai consideravelmente no intervalo de 20 anos. 


\section{GRÁFICO 4}

Índice local segregation para o grupo de 26 a 35 anos das coortes de nascidos entre 1960 e 1969 e 1980 e 1989 (índice 1)

Brasil - 1995-2015

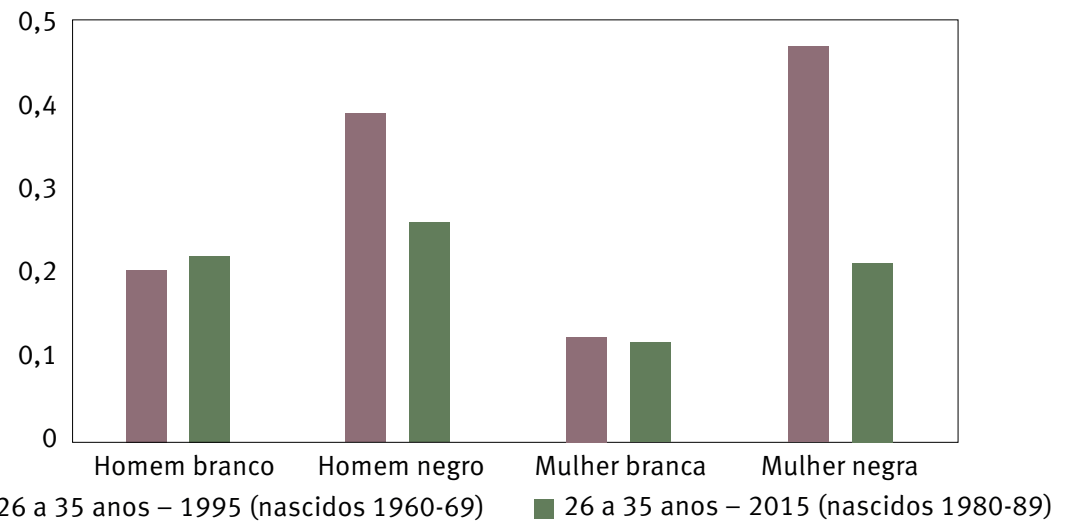

Fonte: IBGE. PNAD 1995 e 2015.

0 mesmo comportamento é observado no Gráfico 5, porém com menor intensidade. 0 grupo dos homens negros, principalmente, experimenta uma queda no índice de 0,37 para 0,29 entre 2005 e 2015, considerando-se, porém, que se trata de um intervalo de tempo menor do que o do Gráfico 4.

\section{GRÁFICO 5}

Índice local segregation para o grupo de 36 a 45 anos das coortes de nascidos entre 1960 e 1969 e 1970 e 1979 (índice 1)

Brasil - 2005-2015

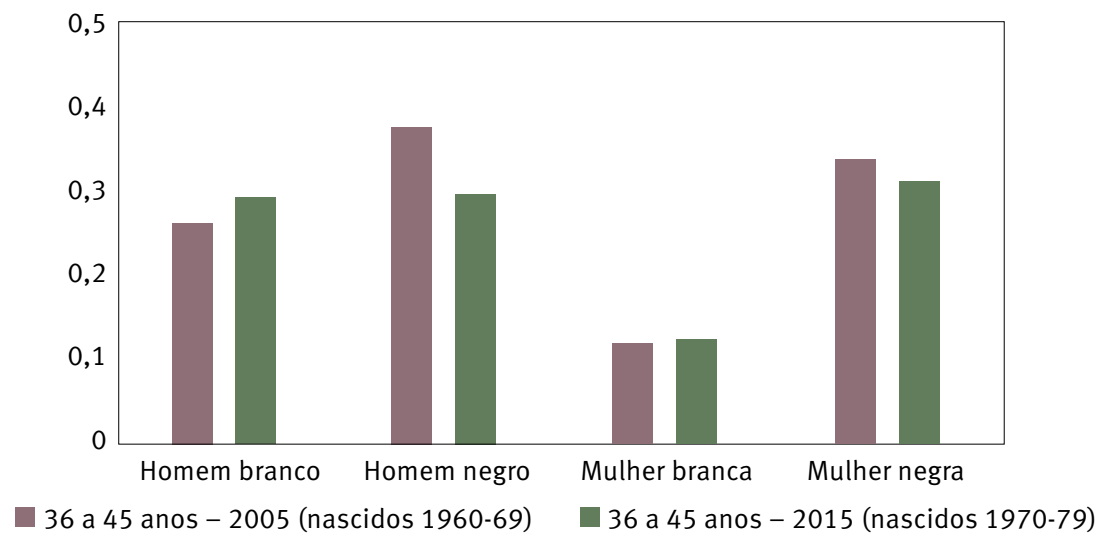

Fonte: IBGE. PNAD 2005 e 2015.

Por último, analisa-se o índice $2(M)$, apresentado na Tabela do Anexo, que sintetiza um valor global para a segregação dos grupos. Os resultados apontam uma tendência de incremento do índice global de segregação por idade, independentemente da coorte. Não se pode dizer que o grupo de trabalhadores nascidos entre 1960 e 1969 possua características 
intrínsecas que levem a uma maior segregação do que a mesma faixa etária de 2015. Em uma visão geral, o que se depreende é que a segregação é maior no intervalo etário de 46 a 55 anos, sinalizando mais um efeito de idade do que um efeito de coorte.

Portanto, a comparação da coorte de nascidos entre 1960 e 1969 tem dois resultados principais: o efeito de idade é mais sobressalente do que o de coorte, ao comparar com os mesmos grupos etários em 2015; e, embora haja pouca variação para o recorte de gênero, para cor/raça os dados apontam uma menor segregação em 2015, principalmente para o grupo de 26 a 35 anos.

\section{Discussão e conclusão}

0 presente artigo se debruçou sobre duas questões: se a segregação ocupacional e a discriminação salarial variam entre recortes etários com ensino superior; e se estes índices eram equivalentes para a coorte nascida entre 1960 e 1969 quando estes indivíduos possuíam de 26 a 35 anos e de 36 a 45 anos - ou seja, em 1995 e 2005, respectivamente. Os resultados revelam que, com relação à primeira pergunta, tais índices de fato são menores para o grupo de 26 a 35 anos em comparação ao de 46 a 55 anos em 2015. Porém, quando explorados os resultados da coorte nascida entre 1960 e 1969, observa-se que estes indivíduos também eram menos segregados.

Primeiramente, realizou-se uma análise de segregação e diferencial salarial por cortes etários (26-35, 36-45 e 46-55 anos) de pessoas com ensino superior completo. Com base em resultados anteriores (SILVEIRA; LEÃO, 2020), verificou-se que a estrutura ocupacional no Brasil se tornou menos segregada em anos mais recentes. Nesse sentido, os índices 1 (Gráfico 2) e 2 demonstram que, de fato, a segregação é menor entre os mais jovens. Isso se reforça no Gráfico 3, em que se observa que a desvantagem salarial é menor entre os mais jovens, embora o termo relativo à discriminação seja persistente em qualquer grupo etário.

Sem realizar recortes etários, o estudo anterior demonstra que, entre 1986 e 2015, o Brasil se tornou ligeiramente menos segregado, sendo que, sobre a renda, o termo atribuído à segregação diminuiu por cor/raça e gênero. Já a discriminação contra as mulheres brancas diminuiu pouco e, para negras e negros, esse percentual até aumentou em alguns cenários, principalmente nos mais elitizados (SILVEIRA; LEÃO, 2020). Os cortes etários contribuem para a compreensão de que essas variações não são homogêneas dentro da estrutura etária brasileira, uma vez que os diferenciais salariais são maiores entre os mais velhos.

Por um lado, os resultados da análise revelam que a segregação está associada à idade. Os nascidos entre 1960 e 1969 são mais segregados aos 50 anos de idade (em 2015) do que aos 30 anos (em 1995). Em 1995 eles possuíam índices gerais (índice 2, M, em especial) semelhantes àqueles do grupo de 26 a 35 anos em 2015. Por outro lado, a coorte 1960-69 tem mudanças significativas desveladas pela análise. Em 1995, a segregação de trabalhadoras e trabalhadores negros era maior do que em 2015, considerando-se a mesma faixa etária. 
Sintetizam-se, então, os achados da análise: a segregação ocupacional por gênero e raça no Brasil é associada à idade, sendo maior entre indivíduos em idades mais avançadas; apesar disso, um efeito de coorte é observado ao se compararem as coortes de nascidos entre 1980 e 1989 e entre 1960 e 1969 no que se refere à cor/raça.

Com relação ao primeiro ponto, no entanto, os resultados encontrados destoam, por exemplo, de Reis e Crespo (2015). Segundo estes autores, a discriminação seria menor entre os mais jovens, uma vez que a idade aumentaria a exposição dos trabalhadores ao mercado, diminuindo as incertezas e, consequentemente, as distorções salariais. Aqui, porém, observou-se que, no caso de mulheres e homens com ensino superior, os resultados são distintos. Para as mulheres negras, o termo de discriminação diminui em $4 \%$ e, para os homens negros, aumenta em $12 \%$ nos grupos etários mais velhos. Já o diferencial salarial dado pela segregação piora com o tempo para os negros de ambos os sexos. Os homens veem sua vantagem com relação à média cair de $12 \%$ para $3 \%$ e a das mulheres passa de $-14 \%$ para $-23 \%$. Então, nesse sentido, o aumento de idade tem contornos e retornos negativos para o grupo negro.

Para além dos mecanismos discriminatórios, olha-se também para os mecanismos institucionais que recaem sobre os grupos etários. Bronson e Thoursie (2017), por exemplo, demonstram que, ao longo do tempo, as promoções são mais favoráveis aos homens. As evidências encontradas para o Brasil mostram relação positiva entre idade e segregação, e idade e discriminação, de maneira que a coorte mais velha possui maiores diferenciais e maior discriminação, principalmente sobre as mulheres negras. Além disso, em geral, às mulheres são aplicadas penalidades referentes à maternidade, que interferem em seus padrões de inserção e rendimentos no mercado de trabalho (GUIGINSKI; WAJNMAN, 2019) - embora, segundo Muniz e Veneroso (2019), as penalidades são mais sensíveis entre as mulheres mais pobres, que fogem ao perfil abordado neste trabalho.

O segundo ponto apresenta dois lados da mesma moeda: por um lado, as coortes mais novas têm uma melhora nos índices de segregação para trabalhadores negros, indicando que estes estão mais bem distribuídos. Isso pode sinalizar que a expansão do ensino superior e as políticas de ações afirmativas têm habilitado mais indivíduos a se inserirem em tais ocupações (MARTELETO et al., 2016). Por outro lado, é importante ressaltar que, embora os índices de inserção sejam melhores entre os mais jovens em 2015, a discriminação é persistente em todos os recortes etários, indicando que o racismo perpassa todos os grupos, de maneira que o elitismo da discriminação permanece rígido (SOARES, 2000; CAMPANTE et al., 2004; SANTOS, 2005).

Estes resultados, ainda, são condizentes com o que foi apontado por Reis e Crespo (2015) e Barbosa (2016). Em ambos os trabalhos, as coortes mais jovens são associadas a cenários de menor desigualdade de renda e menor discriminação por cor/raça, respectivamente. Como sustenta Barbosa (2016), a diminuição da desigualdade de renda entre os homens foi mais intensa naquelas coortes que foram expostas à expansão do ensino básico no país. Para os resultados do presente trabalho, observa-se que as coortes mais 
jovens apresentam menores níveis de segregação ocupacional, o que condiz com um grupo com oportunidades mais bem distribuídas, principalmente considerando que se trata de um recorte restrito àqueles com ensino superior.

Por fim, respondendo às principais perguntas elencadas na seção teórica, os resultados levam às conclusões apontadas a seguir. Por idade, a segregação é menor entre os mais jovens. A questão é: como é possível manter esse nível de segregação mais baixo, ou até mesmo diminuí-lo? Segundo resultados de Bronson e Thoursie (2017), processos internos ao mercado de trabalho diferenciam ainda mais as trajetórias femininas e masculinas, por meio de promoções. Os processos institucionais se tornam centrais na discussão sobre perpetuação das desigualdades. Por período e coorte, pode-se assinalar que os recortes etários mais jovens perpassaram por cenários (político, social e/ou econômico) favoráveis no que tange à situação de trabalhadoras e trabalhadores negros nos anos mais recentes. Os motivos podem ser combinados, como, por exemplo, o aumento da escolaridade média da população em geral, a expansão do ensino superior, o aumento das taxas de formalização a partir dos anos 2000 e até mesmo mudanças culturais para um ambiente mais diversificado e democrático.

As conclusões do artigo apontam, portanto, para o efeito da idade sobre os índices de segregação e como a cor/raça possui variações por coorte. Contudo, há questões em aberto, como aspectos macroeconômicos ou estruturais que podem alterar a desigualdade por gênero e cor/raça; ou quais mecanismos aumentam ou diminuem a distância socioeconômica entre eles? Ao buscar destrinchar tais mecanismos, estratégias de combate às desigualdades podem ser estabelecidas para um futuro próximo.

\section{Referências}

BAILEY, S.; LOVEMAN, M.; MUNIZ, J. Measures of "race" and the analysis of racial inequality in Brazil. Social Sciences Research, v. 42, n. 1, p. 106-119, 2013. DOI: https://doi.org/10.1016/j. ssresearch.2012.06.006.

BARBOSA, R. Comparabilidade das informações disponíveis nos Censos (1960-2010) e PNADS (1976, 1985, 1995 e 2005). São Paulo: Centro de Estudos da Metrópole, 2014. (Texto para Discussão, n. 017/2014). Disponível em: http://centrodametropole.fflch.usp.br/pt-br/ publicacoes/artigos-e-working-papers?artigos=rog\%C3\%A9rio+barbosa\&categoria=All\&ite ms_per_page $=30$.

BARBOSA, R. Desigualdade de rendimentos do trabalho no curto e no longo prazo: tendências de idade, período e coorte. Dados, v. 59, n. 2, p. 385-425, 2016. DOI: https://doi. org/10.1590/00115258201681.

BECKER, G. The economics of discrimination. Chicago: University of Chicago Press, 1957.

BOTASSIO, D.; VAZ, D. Segregação ocupacional por sexo no mercado de trabalho brasileiro: uma análise de decomposição para o período 2004-2015. Revista Brasileira de Estudos de População, v. 37, 2020. DOI: https://doi.org/10.20947/S0102-3098a0131.

BRONSON, M.; THOURSIE, P. The lifecycle wage growth of men and women: explaining gender differences in wage trajectories. Georgetown University, Department of Economics, 2017. (Working Paper). Disponível em: https://ideas.repec.org/p/geo/guwopa/gueconwpa 17-17-06.html. 
BRUSCHINI, C. Gênero e trabalho no Brasil: novas conquistas ou persistência da discriminação? In: ROCHA, M. I. B. da (org.). Trabalho e gênero: mudanças, permanências e desafios. São Paulo: Editora 34, 2000.

BRUSCHINI, C.; LOMBARDI, M. R. Médicas, arquitetas, advogadas e engenheiras: mulheres em carreiras profissionais de prestígio. Estudos Feministas, ano 3, p. 9-24, 1999. DOI: https://doi. org/10.1590/\%25x.

CAMPANTE, F.; CRESPO, A.; LEITE, P. Desigualdade salarial entre raças no mercado de trabalho urbano brasileiro: aspectos regionais. Revista Brasileira de Economia, v. 58, n. 2, p. 185-210, 2004. DOI: https://doi.org/10.1590/S0034-71402004000200003.

CARVALHAES, F. A tipologia Erikson-Goldthorpe-Portocarero (EGP): uma avaliação analítica e empírica. Revista Sociedade e Estado, v. 30, n. 3, p. 673-703, 2015. DOI: https://doi.org/10.1590/ S0102-69922015.00030005.

COSTA RIBEIRO, C. Desigualdades de gênero no ensino superior e no mercado de trabalho no Brasil: uma análise de idade, período e coorte. Revista Sociedade e Estado, v. 31, n. 2, p. 301-323, 2016. Disponível em: http://dx.doi.org/10.1590/S0102-69922016000200002.

DEL RÍO, C.; ALONSO-VILLAR, O. The evolution of occupational segregation in the United States, 1940-2010: gains and losses of gender-race/ ethnicity groups. Demography, v. 52, p. 967-988, 2015. DOI: https://doi.org/10.1007/s13524-015-0390-5.

ENGLAND, P. Devaluation and the pay of the comparable male and female occupations. In: GRUSKY, D. (ed.). Social stratification: class, race, and gender in sociological perspective. Westview Press, 2008.

GUIGINSKI, J.; WAJNMAN, S. A penalidade da maternidade: participação e qualidade da inserção no mercado de trabalho das mulheres com filhos. Revista Brasileira de Estudos de População, v. 36, p. 1-26, 2019. DOI: https://doi.org/10.20947/s0102-3098a0090.

GUIMARÃES, N.; HIRATA, H.; SUGITA, K. Cuidado e cuidadoras: o trabalho de care no Brasil, França e Japão. Sociologia \& Antropologia, v. 1, n. 1, p. 151-180, 2011. DOI: https://doi.org/10.1590/2238$38752011 \mathrm{v} 117$.

HIRATA, H.; KERGOAT, D. Novas configurações da divisão sexual do trabalho. Cadernos de Pesquisa, v. 37, n. 132, p. 595-609, dez. 2007.

KERGOAT, D. Dinâmicas e consubstancialidade das relações sociais. Novos Estudos, v. 86, 2010. DOI: http://dx.doi.org/10.1590/S0101-33002010000100005.

KON, A. Mudanças recentes no perfil da distribuição ocupacional da população brasileira. Revista Brasileira de Estudos de População, v. 23, n. 2, p. 247-267, 2006. DOI: https://doi.org/10.1590/ S0102-30982006000200004.

KORENMAN, S.; NEUMARK, D. Cohort crowding and youth labor markets: a cross-national analysis. Cambridge, MA: National Bureau of Economic Research, 1997. (NBER Working Paper, 6031).

MARTELETO, L.; MARSCHNER, M.; CARVALHAES, F. Educational stratification after a decade of reforms on higher education access in Brazil. Research in Social Stratification and Mobility, v. 46, p. 99-111, 2016. DOI: https://doi.org/10.1016/j.rssm.2016.08.004.

MUNIZ, J.; VENEROSO, C. Diferenciais de participação laboral e rendimento por gênero e classes de renda: uma investigação sobre o ônus da maternidade no Brasil. Dados, v. 62, n. 1, 2019. DOI: https://doi.org/10.1590/001152582019169.

OLIVEIRA, A.; RIOS-NETO, E. Modelos idade-período-coorte aplicados à participação na força de trabalho: em busca de uma versão parcimoniosa. Revista Brasileira de Estudos de População, v. 21, n. 1, p. 21-47, 2004. Disponível em: https://www.rebep.org.br/revista/article/view/280. 
OLIVEIRA, A.; RIOS-NETO, E. Tendências da desigualdade salarial para coortes de mulheres brancas e negras no Brasil. Estudos Econômicos, v. 36, n. 2, p. 205-236, 2006. DOI: https://doi. org/10.1590/S0101-41612006000200001.

PARSONS, T. The social system. Londres: Tavistock, 1951.

PRATES, I. 0 sistema de profissões no Brasil: formação, expansão e fragmentação. Um estudo de estratificação social. Tese (Doutorado em Sociologia) - Universidade de São Paulo (USP), São Paulo, 2018.

REIS, M.; CRESPO, A. Race discrimination in Brazil: an analysis of the age, period and cohort effects. Brasília; Rio de Janeiro: Ipea, 2015. (Discussion Paper, 157).

RIOS-NETO, E.; OLIVEIRA, A. M. Aplicação de um modelo de idade-período-coorte para a atividade econômica no Brasil metropolitano. Pesquisa e Planejamento Econômico, v. 29, n. 2, p. 243-272, 1999. Disponivel em: http://repositorio.ipea.gov.br/handle/11058/5033.

SANTOS, J. Efeitos de classe na desigualdade racial no Brasil. Dados, v. 48, n. 1, p. 21-65, 2005. DOI: https://doi.org/10.1590/S0011-52582005000100003.

SANTOS, J. Classe social e desigualdade de gênero no brasil. Dados, v. 51, n. 2, p. 355-402, 2008. DOI: https://doi.org/10.1590/S0011-52582008000200005.

SILVA, N. Diferenciais raciais de rendimento. In: HASENBALG, C.; SILVA, N.; LIMA, M. Cor e estratificação social. Rio de Janeiro: Contra Capa Livraria, 1999.

SILVEIRA, L.; LEÃO, N. O impacto da segregação ocupacional por gênero e raça na desigualdade de renda no Brasil em três décadas (1986-2015). Revista Latinoamericana de Población, v. 14, n. 27, 2020. DOI: https://doi.org/10.31406/relap2020.v14.i12.n27.2.

SOARES, S. O perfil da discriminação no mercado de trabalho - homens negros, mulheres brancas e mulheres negras. Brasília: Ipea, 2000. (Texto para Discussão, n. 769).

\section{Sobre os autores}

Leonardo Souza Silveira é doutor em Sociologia pela Universidade Federal de Minas Gerais (UFMG) e bolsista PNPD no Instituto de Pesquisa Econômica Aplicada (Ipea), Rio de Janeiro-RJ.

Natália Leão Siqueira é doutoranda em Sociologia pelo Instituto de Estudos Sociais e Políticas da Universidade Estadual do Rio de Janeiro (Iesp-UERJ).

\section{Endereço para correspondência}

Leonardo Souza Silveira

Avenida Presidente Vargas, $730,17^{\circ}$ andar, Centro

20071-000 - Rio de Janeiro-RJ, Brasil

Natália Leão Siqueira

Rua da Matriz, 82, Botafogo

22260-100 - Rio de Janeiro-RJ, Brasil 


\begin{abstract}
Occupational segregation and income gap by gender and race in Brazil: an age group analysis
\end{abstract}

In the past 30 years, Brazil strengthened labor market structural change on aspects related to occupational modernization, feminine participation and Higher Education expansion. In this regard, younger cohorts entered in a different context than those who entered in past decades. Thus, this research aims to measure occupational segregation and wage disparities by gender and race over three age groups. Therefore, the questions are: Is occupational segregation lower among younger people? Does it reflect on lower wage inequality? These questions are analyzed through segregation and wage decomposition from PNAD 2015 on a sample for people with Higher Education. These results point out to lower inequality among the youngest people, but doubts remain as to whether these are age or cohort effects. In this regard, the segregation index for the cohort between 26 and 35 years old in 2015 is compared to the same age range in 1995 and 2005 , focusing on isolated cohort and age effects. In the end, it is pointed out that age is more associated with increasing inequality than with cohort, although segregation by race is lower among the younger cohort.

Keywords: Occupation segregation. Occupations. Gender. Race. Inequality.

\title{
Resumen
}

Segregación ocupacional y diferenciales de ingresos por género y raza en Brasil: un análisis de grupos de edad

En los últimos treinta años, Brasil ha consolidado aspectos de cambio estructural en el mercado laboral relacionados con la modernización ocupacional, la participación femenina y la expansión de la educación superior. En este sentido, las cohortes más jóvenes se insertan en un contexto diferente del que se insertaron en décadas pasadas. Por tanto, la presente investigación tiene como objetivo medir la variación en la segregación ocupacional y la brecha salarial por género y raza en tres grupos de edad. En este sentido, las preguntas son: ¿La segregación ocupacional por género y raza es menor entre las personas más jóvenes? ¿Se refleja esto en una menor desigualdad salarial? Estas preguntas se analizan mediante índices de segregación y descomposiciones del salario basados en el PNAD de 2015 en una muestra de personas con estudios superiores completos. Los resultados muestran tasas más bajas de desigualdad entre las personas más jóvenes, pero aún dejan dudas sobre si son efectos de la edad o la cohorte. Para esto, las tasas de segregación de la cohorte de entre 26 y 35 años en 2015 se comparan con el mismo intervalo en los años 1995 y 2005, para separar los efectos de cohorte y edad. Al final, se indica que la edad está más asociada con una mayor desigualdad que la cohorte, aunque entre las personas más jóvenes hay menos segregación por raza.

Palabras clave: Segregación ocupacional. Ocupaciones. Género. Raza. Desigualdad. 


\section{Anexo}

Índices de local measure segregation, $M$, contribuição do grupo, decomposição entre e intragrupos, por grupos etários e coortes

Brasil - 1995-2015

\begin{tabular}{cccccc}
\hline & 2015 & 2005 & 1995 & $\begin{array}{c}\text { Coorte de } \\
\text { nascidos entre }\end{array}$ & $\begin{array}{c}\text { Coorte de } \\
\text { nascidos entre }\end{array}$ \\
\cline { 2 - 5 } & $\begin{array}{c}\text { Grupo } 1 \\
\text { I26 a 35 anos) }\end{array}$ & $\begin{array}{c}\text { Grupo } 2 \\
\text { (36 a 45 anos) }\end{array}$ & $\begin{array}{c}\text { Grupo } 3 \\
\text { (46 a 55 anos) }\end{array}$ & $\begin{array}{c}1960-69 \\
\text { (36 a 45anos) }\end{array}$ & $\begin{array}{c}\text { 1960-69 } \\
\text { (26 a 35 anos) }\end{array}$ \\
\hline
\end{tabular}

\begin{tabular}{|c|c|c|c|c|c|}
\hline \multicolumn{6}{|c|}{$\begin{array}{l}\text { Índice } 1 \text { - local } \\
\text { measure segregation }\end{array}$} \\
\hline Homem branco & 0,2223 & 0,2909 & 0,3283 & 0,2572 & 0,2021 \\
\hline Mulher branca & 0,1206 & 0,1236 & 0,1623 & 0,1204 & 0,1281 \\
\hline Homem negro & 0,2587 & 0,2926 & 0,4099 & 0,3732 & 0,3912 \\
\hline Mulher negra & 0,2121 & 0,3092 & 0,3341 & 0,3344 & 0,4720 \\
\hline \multicolumn{6}{|l|}{ Índice $2-M$} \\
\hline$M$ & 0,1889 & 0,2386 & 0,2827 & 0,2236 & 0,2137 \\
\hline Sexo & 0,1327 & 0,1672 & 0,2011 & 0,1614 & 0,1497 \\
\hline Cor/raça & 0,0393 & 0,0543 & 0,0639 & 0,0490 & 0,0469 \\
\hline \multicolumn{6}{|c|}{$\begin{array}{l}\text { Índice } 3 \text { - } \\
\text { contribuição do grupo }\end{array}$} \\
\hline Homem branco & 0,3267 & 0,3337 & 0,3226 & & \\
\hline Mulher branca & 0,2312 & 0,1755 & 0,2066 & & \\
\hline Homem negro & 0,2083 & 0,1973 & 0,2306 & & \\
\hline \multirow[t]{2}{*}{ Mulher negra } & 0,2335 & 0,2933 & 0,2401 & & \\
\hline & & 2015 & & & \\
\hline \multicolumn{6}{|c|}{$\begin{array}{l}\text { Índice } 4 \text { - } \\
\text { Decomposição salarial } \\
\text { entre ocupações }\end{array}$} \\
\hline Homem branco & 0,1644 & 0,2049 & 0,2167 & & \\
\hline Mulher branca & $-0,0947$ & $-0,0427$ & $-0,0622$ & & \\
\hline Homem negro & 0,1210 & 0,0264 & 0,0374 & & \\
\hline Mulher negra & $-0,1430$ & $-0,2027$ & $-0,2348$ & & \\
\hline \multicolumn{6}{|c|}{$\begin{array}{l}\text { Índice } 5 \text { - } \\
\text { discriminação salarial } \\
\text { na mesma ocupação }\end{array}$} \\
\hline Homem branco & 0,2164 & 0,1117 & 0,2705 & & \\
\hline Mulher branca & $-0,0476$ & 0,0723 & $-0,0622$ & & \\
\hline Homem negro & $-0,0655$ & $-0,1184$ & $-0,1903$ & & \\
\hline Mulher negra & $-0,1579$ & $-0,1592$ & $-0,1105$ & & \\
\hline
\end{tabular}

Fonte: IBGE. PNAD 1995, 2005 e 2015. 